\title{
Evaluación de los procesos superficiales de escorrentía en cárcavas originadas en olivares convencionales. Un apartado a tener en cuenta en la planificación territorial
}

\author{
Evaluation of surface runoff processes in gullies \\ originated in conventional olive groves. A section to \\ consider in territorial planning
}

\author{
Jesús Rodrigo-Comino1, Encarnación V. Taguas², Manuel K. Seeger ${ }^{3}$ \\ y Johannes B. Ries ${ }^{4}$
}

\begin{abstract}
RESUMEN
Se analiza la respuesta hidrológica de una cárcava efímera frente a un evento de lluvia equivalente de frecuencia "ordinaria" ( $\approx 1$ año) a partir de una simulación de escorrentía en una cuenca experimental con olivar en Puente Genil, Córdoba (España). Mil litros de agua fueron bombeados durante 10 minutos y la descarga total, la velocidad superficial de avance de frente de flujo, la concentración de sedimentos y los cambios morfológicos en el perfil transversal fueron calculados. Los resultados mostraron elevadas diferencias entre las tasas de escorrentía de hasta un 17\% entre las condiciones "secas" y "húmedas". La concentración de sedimentos instantánea llegó a alcanzar valores de hasta $40 \mathrm{~g} \mathrm{l}^{-1}$. Se concluye que las cárcavas en olivares convencionales son capaces de registrar elevadas tasas de erosión y escorrentía, particularmente bajo condiciones de mayor humedad. Se requiere más información y atención a las medidas de control sobre la erosión por el flujo concentrado de la escorrentía en la planificación agrícola.
\end{abstract}

Palabras clave: Olivar; erosión; cárcava efímera; planificación agrícola; simulación de escorrentía.

\begin{abstract}
The hydrological response of one ephemeral gully during an "ordinary" rainfall event (with a return period of approximately 1 year) was assessed using a runoff simulation in an experimental catchment cultivated with olive groves in Puente Genil, Córdoba (Spain). 1000 I of water were pumped for 10 minutes in order to measure the total discharge, flow front velocity, sediment concentration and morphological changes of the transversal profile. The results showed high differences among runoff coefficients (of about 17\%) under "dry" and "wet" conditions. The instantaneous sediment concentration values up to $40 \mathrm{~g} \mathrm{I}^{-1}$. It was concluded that the ephemeral gullies in conventional olive groves are able to register high soil and water losses, particularly, under higher wet conditions. Thus, the importance to collect more information and pay attention to the soil erosion control measures against the concentrated runoff in agricultural land management plans have to be addressed.
\end{abstract}

Key words: Olive orchards; erosion; ephemeral gullies; agricultural management; runoff simulation.

Department of Physical Geography, University of Trier, 54296 Trier, Germany. Soil Erosion and Degradation Research Group, Department of Geography, University of Valencia, Avda. Blasco Ibáñez, 28, 46010 Valencia, Spain 


\section{Introducción}

La conservación del suelo es un tema primordial dentro de la ordenación y gestión del territorio, siendo vital tanto en usos agrarios como naturales (García-Ruiz et al. 2013; Martínez-Casasnovas y Sánchez-Bosch 2000). Sin embargo, debido a la elevada sobreexplotación del recurso suelo, claros procesos erosivos están siendo detectados en forma de transporte de sedimentos, agua, nutrientes y contaminantes a través de regueros, cárcavas o canales artificiales (Ben Slimane et al. 2015; Marzolff y Poesen 2009).

Especialmente, en toda la franja mediterránea en campos cultivados con olivar convencional, esta problemática se está convirtiendo en un debate entre investigadores, agricultores y gestores del territorio (Fleskens y Stroosnijder 2007; Gómez et al. 2008). En Italia, específicamente en el norte de la región de Calabria, Conforti et al., (2013) cuantificaron para un cuenca con olivares y con problemas de generación de regueros y cárcavas tasas de erosión mayores a $6 \mathrm{t} \mathrm{ha}^{-1} \mathrm{a}^{-1}$. Para olivares griegos en el centro de Creta, Kairis et al., (2013) registraron una pérdida anual de 3,4 $\mathrm{mm} \mathrm{a}^{-1}$ en parcelas experimentales. En Jordania, Kraushaar et al., (2014), utilizando el método de los tocones del olivo, llegaron a registrar hasta $95 \mathrm{t}^{-1} \mathrm{ha}^{-1} \mathrm{a}^{-1}$. Por ejemplo, en el sur de España, Parras-Alcántara et al., (2016) estimaron 6,6 t ha-1 a-1 para olivares convencionales utilizando la RUSLE (Revised Universal Soil Loss Equation).

En el caso de los olivares españoles, numerosos son los estudios publicados en Andalucía (Sur de España), que estiman un total de 1,52 millones de ha cultivadas, con una producción media entre las campañas 2006/07 y 2013/14 de 5,48 millones de toneladas (Plan Director Andaluz del Olivar). Específicamente en Córdoba, los olivares convencionales localizados en áreas de cierta pendiente, que mantienen buena parte de la superficie de sus suelos desnudos y en el que los agricultores utilizan maquinaria para eliminar la cobertura vegetal, están registrando serios problemas de hidrofobicidad, compactación y reducción del horizonte fértil (Burguet et al. 2016; Di Stefano et al. 2016). Vanwalleghem et al., (2010), registraron utilizando el método del tocón del olivo como bioindicador pasivo de los cambios de altura del nivel del suelo, una erosión comprendida entre 70 y $100 \mathrm{t} \mathrm{ha}^{-1} \mathrm{a}^{-1}$, estimando además como máximos históricos locales valores de más de $180 \mathrm{t} \mathrm{ha}^{-1} \mathrm{a}^{-1}$. A través del método del número de curva desarrollado para estimar de forma empírica la escorrentía superficial de acuerdo al tipo de suelo, uso, manejo y condición hidrológica, Taguas et al., (2015a) llegaron a demostrar que las tasas de escorrentía potenciales deberían ser tenidas en cuenta para la planificación territorial debido a la recurrencia de los eventos de lluvia muy erosivos. También, Taguas et al., $(2013,2015 b)$ tras la monitorización de regueros y cárcavas en la misma zona, a nivel de cuenca detectaron tasas de escorrentía por eventos superiores a 7,2 y 17,0 t para un período de retorno de tres años. De esta forma, el uso de sistemas de cobertura vegetal o del laboreo planificado para frenar la erosión, deberían ser medidas obligatorias más allá de las obligaciones derivadas de condicionalidad de la Política Agraria Común, o incluso, subsidiadas como muchos agricultores reclaman también en España (Rodrigo-Comino et al. 2019; Franco Martínez y Rodríguez Entrena 2009), para frenar estos procesos de degradación que, por otra parte, podrían ser irreparables a medio-largo plazo en el olivar (Gómez et al. 1999; 2009)

Ante las perspectivas de un inminente cambio en las temperaturas y distribución de las precipitaciones a lo largo del año según los paneles de estudio del cambio climático (Smith 2018), las investigaciones que consigan demostrar que bajo condiciones ordinarias que la erosión es un problema a tener en cuenta, tendrán una mayor repercusión a la hora de convencer a los gestores 
del territorio y agricultores para implementar medidas útiles de conservación del suelo. Para alcanzar este objetivo, es de vital importancia recalcar el papel negativo que juegan las cárcavas en la erosión del suelo.

Para demostrar dicha afirmación, las simulaciones de escorrentía conforman un método poco explotado en la literatura sobre suelos y geografía física en España y en español. Al igual que las simulaciones de lluvia, los experimentos de simulación de escorrentía permiten evaluar y cuantificar bajo condiciones hipotéticas, reproducibles en otros territorios y controladas por el investigador, múltiples parámetros hidrológicos y erosivos (García-Díaz et al. 2017; Kavian et al. 2017) lo cual permite complementar estudios realizados en laboratorio y que resultan de gran utilidad para el diseño o evaluación de modelos hidrológicos. En el caso de los procesos erosivos de flujo concentrado, puede citarse por ejemplo el modelo AnnAGNPS (Bingner et al. 2018). Dicha metodología sirve para cuantificar el avance de cabecera de cárcavas efímeras, las cuales se desarrollan tras un evento o el paso de la maquinaria y suelen tener un carácter temporal y tamaño reducido (Martínez-Casasnovas, Ramos y Balasch 2013; Taguas et al. 2012) y se basa precisamente en ecuaciones desarrolladas en canales de laboratorio hidráulico (Alonso et al. 2002) y donde la información obtenida en campo complementa los hallazgos del modelo.

En el departamento de Geografía Física de la Universidad de Treveris (Renania-Palatinado, Alemania), desde hace una década se comenzó el desarrollo y continuas mejoras de un simulador de escorrentía con objeto de evaluar y cuantificar el comportamiento erosivo e hidrológico de los regueros y las cárcavas (Wirtz et al. 2010). En la actualidad, diversos trabajos han sido publicados en campos abandonados, badlands, viñedos o almendrales (Rodrigo-Comino et al. 2017; Wirtz et al. 2012; 2013). Sin embargo, este tipo de estudios todavía no han sido propiamente llevado a cabo en olivares convencionales para completar sus resultados con observaciones realizadas a otras escalas, como las simulaciones de lluvia en pequeñas parcelas (Rodrigo-Comino et al. 2018), o el análisis del Número de Curva (Taguas et al., 2015a) y las medidas de las relaciones lluvia-escorrentía-descarga de sedimentos a escala de cuenca con estaciones experimentales (Taguas et al., 2013; 2015)

Por lo tanto, el principal objetivo de este estudio fue analizar e interpretar la respuesta hidrológica y erosiva de una cárcava efímera a un evento "ordinario" (frecuencias entre 1 y 2 años de período de retorno) a partir de un experimento de simulación de escorrentía en un olivar marginal con una pendiente media del 15\%, comparando los resultados bajo condiciones contrastadas de humedad ("secas" y "húmedas"). Estos ensayos en campo permiten complementar la información de experimentos similares en condiciones de laboratorio que han permitido la elaboración de modelos predictivos para el análisis de la erosión por flujo concentrado y sirven para evaluar el efecto encadenado de eventos erosivos "frecuentes".

\section{Materiales y métodos}

\section{Área de estudio}

El campo cultivado con olivar convencional donde se encuentra la cárcava que fue sometida a la simulación de escorrentía está localizada en el municipio de Puente Genil, Córdoba (37, 4º 
N, 4, $8^{\circ}$ O). En la Figura N. ${ }^{\circ}$ 1, es posible observar como numerosas formas de erosión de tipo lineal, como regueros o cárcavas efímeras, se reparten por toda la cuenca. Dicha cuenca ocupa una extensión de 6,1 ha con una pendiente media del 15\%, aunque con gradientes que pueden alcanzar el $20 \%$ (y el 100\%). La altitud de la cuenca está comprendida entre 213 y $262 \mathrm{~m}$ sobre el nivel del mar.

La temperatura media diaria anual es de aproximadamente $18^{\circ} \mathrm{C}$, con valores medios máximos que superan los $25^{\circ} \mathrm{C}$. Suele ser común que los promedios más elevados se registren en el mes de julio, siendo los meses de verano julio, agosto de septiembre cuando la precipitación es mínima y se dispara la evapotranspiración y por consiguiente, el déficit hídrico. Por el contrario, las temperaturas diarias más bajas se registran en enero, siendo menores a $10^{\circ} \mathrm{C}$. La precipitación media anual es de unos $400 \mathrm{~mm}$, registrándose los máximos entre los meses de septiembre y enero, con períodos de aridez durante los meses de verano. Taguas et al., (2010) calculó para un período de retorno de 2 años, un valor de lluvia diaria de $43,1 \mathrm{~mm}$. Estos datos, permiten clasificar el área de estudio dentro de un clima típicamente mediterráneo.

Figura $\mathrm{N}^{\circ} 1$.

Localización del área de estudio y mapa geomorfológico de la cuenca seleccionada.

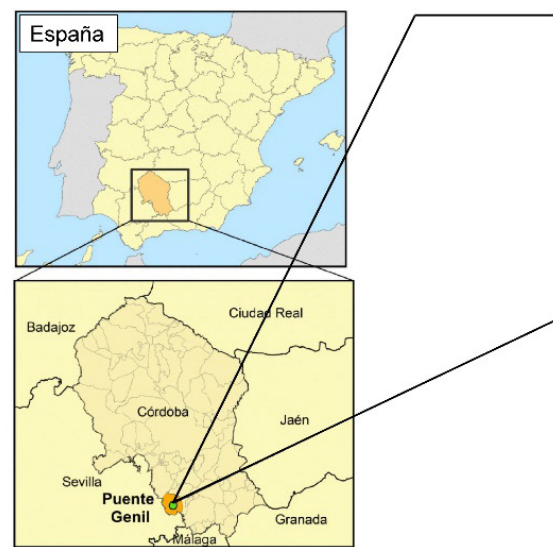

Principales formas de erosión

Lineales

Cárcava

EI....... Reguero rectangular $\longleftarrow$ Cárcava efimera en forma de $U$

-....... Reguero en formar de $\mathrm{V}=$ Escarpe $<1 \mathrm{~m}$

$\ldots$ Reguero en formar de $U$........ Escarpe 1-1,5 m

No lineales

Hethe Sustrato rocoso $\left(>10 \mathrm{~m}^{2}\right)$

Erosión en surcos

Erosión en surcos (>10/10 m)

$\therefore \therefore$ Zonas de acumulación

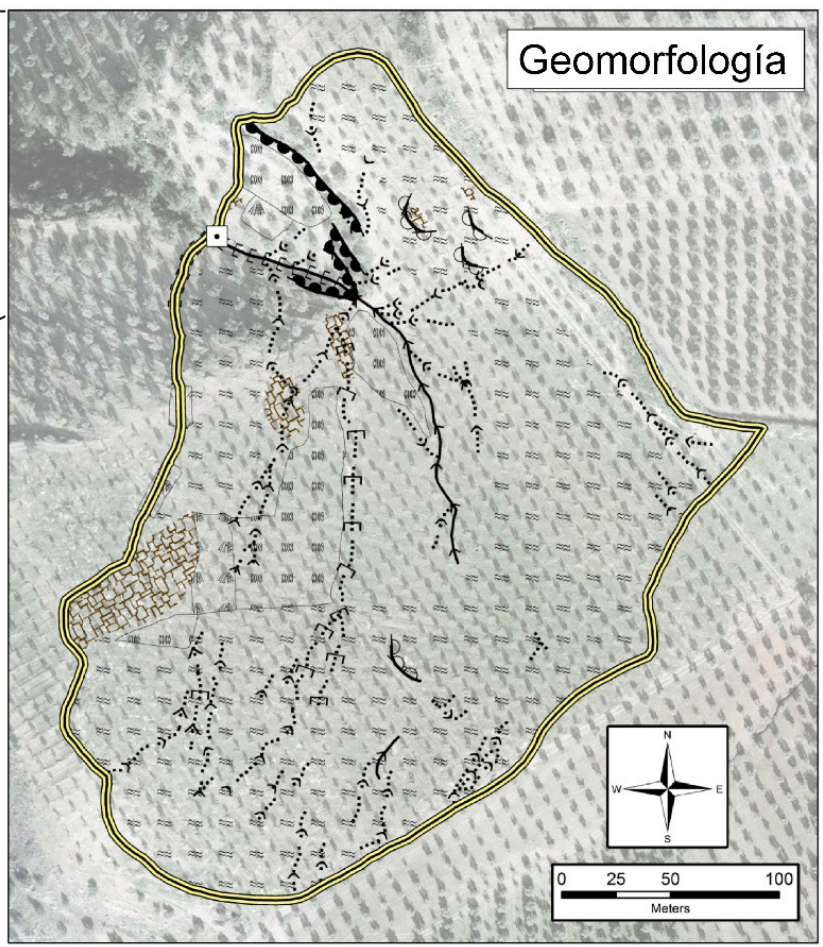

Respecto a la litología, se localizan materiales sedimentarios y, concretamente, calizas que permiten registrar numerosos salientes rocosos que demuestran el rebaje del perfil del suelo y, a su vez, dificultan el laboreo (también cartografiados en la Figura 1). Dicho material da lugar, según Taguas et al., (2013) a suelos arcillo-arenosos (77\% de arenas, $8 \%$ de limos y $15 \%$ de arcillas) con una densidad aparente mayor a $1,6 \mathrm{~g} \mathrm{~cm}^{3}$. El pH es básico, con valores en torno a 8,8. Dichos 
valores son el resultado de un elevado contenido en carbonatos ( $>45 \%)$. Al mantener el suelo sin cobertura vegetal y debido a los elevados contenidos en carbonatos, la materia orgánica en los horizontes superficiales no suele superar el 1,5\%.

El manejo tradicional del suelo está orientado a la obtención de aceituna y aceite de oliva procedentes de la variedad arbequina. La producción anual es baja, pudiendo ser considerada como de carácter extensivo, alcanzando anualmente menos de $2 \mathrm{t} \mathrm{ha-1}$. El suelo no es labrado durante el año, pero la cobertura herbácea espontánea que crece entre las calles es eliminada una o dos veces al año, utilizando maquinaria o herbicidas. Por lo tanto, claros signos de compactación del suelo pueden ser observados en las zonas de rodadas de los tractores.

\section{Experimento de la simulación de escorrentía}

Para llevar a cabo la simulación de escorrentía, una cárcava de 18 metros de longitud fue seleccionada el 19/09/2015. Las condiciones de humedad previa eran en torno al 1-2\%, sin lluvias durante los dos meses anteriores y con temperaturas máximas diarias superiores a los $30^{\circ} \mathrm{C}$. Se trataba de una cárcava efímera de origen natural que responde a los patrones de la dirección de flujo en cuanto a la topografía local se refiere, originada por eventos intensos de lluvia y, por consiguiente, por la generación de escorrentía y transporte de sedimentos en la misma. La pendiente media original es de $8,7^{\circ}$ con un área de desagüe aproximada de $0,6 \mathrm{ha}$, aproximadamente medida sobre un modelo de elevación 1,5 x 1,5 m (Taguas et al. 2013).

El método que se resume en las Figuras 2 y el Cuadro 1 fue estandarizado por el Departamento de Geografía Física en la Universidad de Treveris (Renania-Palatinado, Alemania) para poder ser aplicado bajo las mismas condiciones técnicas que en otras áreas de estudio (Wirtz et al., 2012;2013). Dos repeticiones con una separación de un intervalo de unos 20-25 minutos fueron realizadas, considerando la primera bajo una condición "seca" (A) y la segunda como "húmeda" (B). La primera simulación alcanzó una duración de 9 minutos y la segunda de 10, teniendo en cuenta que fueron bombeados 1000 litros de agua $\left(1 \mathrm{~m}^{3}\right)$ con una intensidad aproximada de 100 a $111 \mathrm{I} . \mathrm{min}^{-1}$. Para ello, se tuvieron en cuenta las relaciones intensidad-duración-frecuencia determinadas en la cuenca y un coeficiente de escorrentía entre 0,05 y 0,10 justificados de la serie de datos adquirida en la cuenca (Taguas et al., 2013). Se evaluaron las frecuencias o períodos de retorno de los caudales generados por el método racional (Chow et al. 1994).

Cuadro 1.

Parámetros para caracterizar el impacto de la generación de escorrentía en la cárcava efímera.

\begin{tabular}{|l|l|l|l|}
\hline Ensayos & Parámetro & \multicolumn{1}{|c|}{ Método y materiales } & $\begin{array}{l}\text { Localización (estaciones) y } \\
\text { período }\end{array}$ \\
\hline \multirow{2}{*}{ Escorrentía } & $\begin{array}{c}\text { Velocidad de } \\
\text { flujo }\left(\mathrm{m} \mathrm{s}^{-1}\right)\end{array}$ & $\begin{array}{l}\text { Medidas de tiempo para } \\
\text { una distancia conocida, ob- } \\
\text { servador, trazador y cronó- } \\
\text { metro }\end{array}$ & $\begin{array}{l}15 \text { puntos separados por 1 } \\
\text { metro; Tiempo de llenado } \\
\text { de botella (3 medidas fren- } \\
\text { te, 3 minutos y 6 minutos } \\
\text { desde el inicio) }\end{array}$ \\
\cline { 2 - 4 } & Caudal $\left(\mathrm{I} \mathrm{s}^{-1}\right)$ & $\begin{array}{l}\text { Medidas de tiempo para un } \\
\text { volumen conocido; botellas } \\
\text { y cronómetro }\end{array}$ & $\begin{array}{l}\text { Salida del de la cárcava; } \\
\text { cada 30 s aproximadamen- } \\
\text { te }\end{array}$ \\
\hline
\end{tabular}




\begin{tabular}{|c|c|c|c|}
\hline Ensayos & Parámetro & Método y materiales & $\begin{array}{c}\text { Localización (estaciones) y } \\
\text { período }\end{array}$ \\
\hline \multirow[b]{2}{*}{$\begin{array}{l}\text { Erosión } \\
\text { (Transporte de } \\
\text { sedimentos) }\end{array}$} & $\begin{array}{l}\text { Concentración } \\
\text { de sedimentos } \\
\qquad\left(\mathrm{g} \mathrm{l}^{-1}\right)\end{array}$ & $\begin{array}{l}\text { Medidas instantáneas de } \\
\text { peso de sedimentos para } \\
\text { un volumen de agua cono- } \\
\text { cido; estufa y recipientes }\end{array}$ & $\begin{array}{l}3 \text { puentes (Fig. } 2 \text { ) a } 5,5,10 \\
\text { y } 17 \mathrm{~m} \text { del inicio - medidas } \\
\text { a } 0 ; 1,3 \text { y } 6 \text { minutos del co- } \\
\text { mienzo del ensayo. }\end{array}$ \\
\hline & $\begin{array}{l}\text { Variación de } \\
\text { las secciones } \\
\text { transversales }(\mathrm{m})\end{array}$ & $\begin{array}{l}\text { Láser instalado en cada } \\
\text { puente (Fig. 2) para medir } \\
\text { el eje transversal de cada } \\
\text { sección cada } 2 \mathrm{~cm} \text { (Figura } \\
\mathrm{N} \cdot{ }^{\circ} \text { e). }\end{array}$ & $\begin{array}{l}\text { Antes de las medidas (A) } \\
\text { bajo condiciones secas; } \\
\text { después de }(A) \text { y antes de } \\
\text { (B); después de las medi- } \\
\text { das bajo condiciones hú- } \\
\text { medas. }\end{array}$ \\
\hline Topografía & $\begin{array}{l}\text { Medidas del } \\
\text { perfil longitudinal } \\
(\mathrm{m})\end{array}$ & $\begin{array}{l}\text { Compás con una capacidad } \\
\text { de abertura de } 1 \text { metro y un } \\
\text { clinómetro digital con } 0,1^{\circ} \\
\text { de precisión. }\end{array}$ & $\begin{array}{l}15 \text { puntos separados por } 1 \\
\text { metro; medidas previas a } \\
\text { las simulaciones. }\end{array}$ \\
\hline
\end{tabular}

Figura N.․ 2 .

Diseño experimental de la simulación de escorrentía. a: depósito de agua; b: bomba de agua; c: comienzo de la cárcava; d: equipo de medición; e: puente de medición; f: medición del caudal.

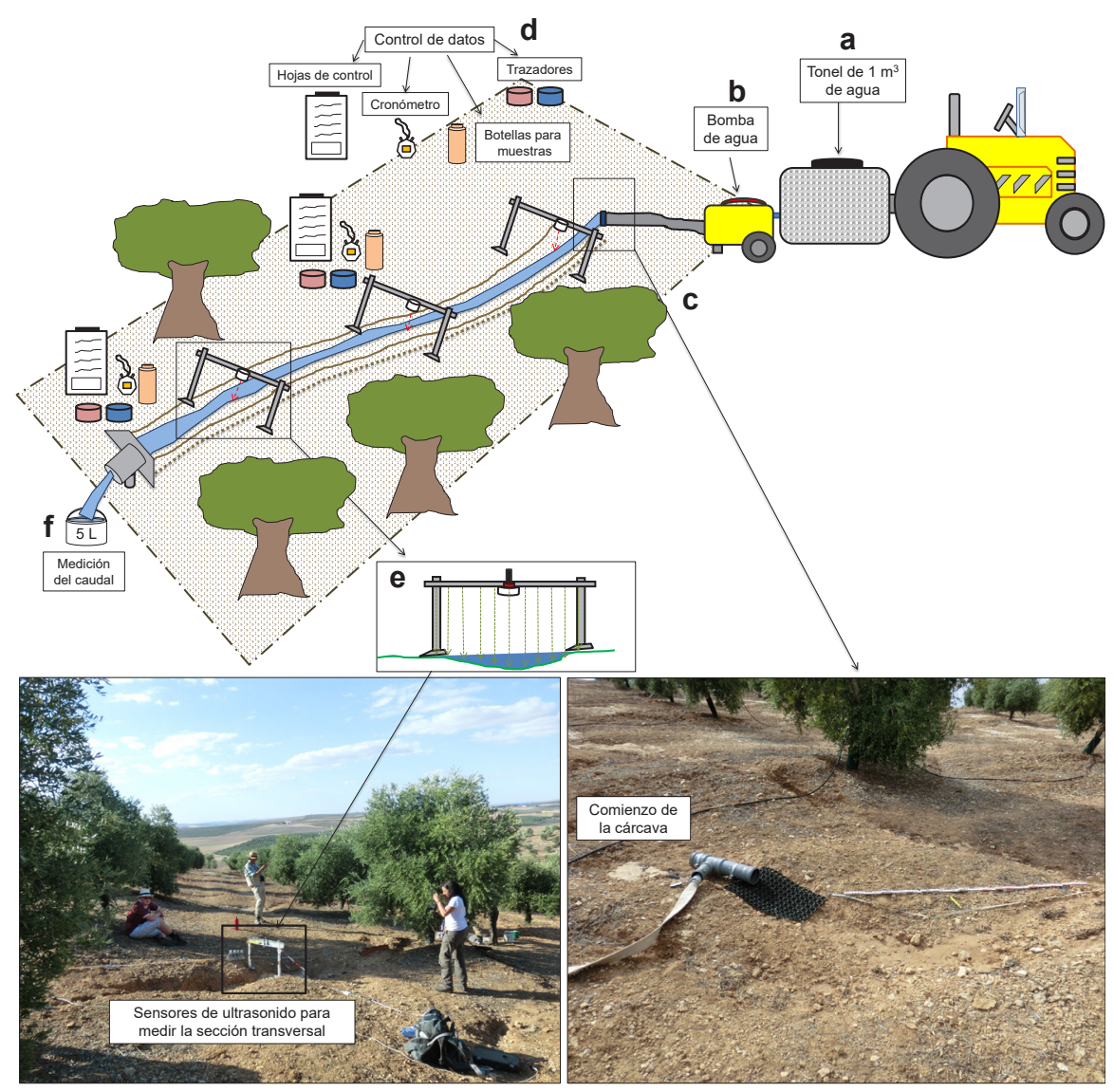


El agua para el experimento se situó en un contenedor de plástico (Figura N. $2 a$ ) y fue bombeada con un motor Honda de potencia constante que suministró valores de caudal entre 0,70 y 1,33 I s-1 durante 12-15 minutos (Figura N. ${ }^{\circ} 2$ b). Al comienzo de la cárcava, una malla de goma se colocó sobre el suelo con objeto de evitar la socavación derivada del impacto del flujo en la base y paredes de la cárcava que incrementaran las medidas de arrastre de sedimentos (Figura N. ${ }^{\circ} 2 \mathrm{C}$ ).

Las medidas de velocidad del flujo de la escorrentía para cada punto, con un paso de aproximadamente $1 \mathrm{~m}$, fueron evaluadas a partir de tres medidas (frente, trazador 1 y trazador 2), utilizando referencias de un metro en las que se cronometraba el avance (Figura N. ${ }^{\circ} 2 \mathrm{~d}$ ). Para ello, al iniciar el experimento se midió el frente del flujo superficial. La segunda medida se realizó transcurridos 3 minutos mientras que la tercera, se llevó a cabo pasados 6 minutos. El uso de trazadores de color azul y rojo permitió diferenciar las segundas y terceras medidas de velocidad. Para todas las mediciones, se utilizaron cronómetros digitales sincronizados.

Tres puentes o puntos de medida del perfil de la cárcava, así como las canalizaciones para las medidas de concentración de sedimentos por el flujo, fueron instalados a 5,5, 10,0 y 17,0 metros, respectivamente. En cada puente, un láser fue instalado para medir el eje transversal de cada sección cada $2 \mathrm{~cm}$ antes y después de la simulación (Figura N. ${ }^{\circ} 2 \mathrm{e}$ ). Antes de llevar a cabo el experimento, se procedió a la medición longitudinal del perfil utilizando un compás con una capacidad de abertura de 1 metro y un inclinómetro digital con 0,10 de precisión. Para facilitar la interpretación de los resultados a lo largo del perfil longitudinal, se procedió también a la medición de los saltos o nickpoints dentro del perfil.

También, se utilizaron botellas de plástico de 1 litro para cuantificar en el laboratorio la concentración de sedimentos $\left(\mathrm{g} \mathrm{l}^{-1}\right)$. Durante el lanzamiento del trazador, transcurridos unos cinco segundos (o cuando la botella se llenaba completamente), fueron introducidas dentro de la corriente de agua generada. Las muestras fueron transportadas al laboratorio e inmediatamente secadas en estufas a $105^{\circ} \mathrm{C}$ durante $24 \mathrm{~h}$ para obtener el peso del sedimento seco movilizado.

Finalmente, una pequeña estación para medir el caudal en el punto de salida de la cárcava fue instalada para poder calcular volumétricamente el caudal desaguado a intervalos regulares.

\section{Tratamiento de los datos y análisis estadísticos}

El análisis de los hidrogramas, los gráficos de velocidad en las distintas estaciones, los valores de concentración instantánea en los tres puentes de aforo, así como la representación de las secciones antes y después de los eventos fueron realizados para cuantificar e ilustrar la respuesta hidrológica a las distintas condiciones de los experimentos.

Para comparar los resultados para el primer evento (A, condiciones secas) y el segundo evento ( $B$, condiciones húmedas), se comprobó la normalidad de la distribución a través del test de Shapiro-Wilk es, y se realizó un ANOVA de una cola para determinar la significancia de sus diferencias. Cuando la distribución no fue normal en ningún caso se realizó el test, y se aplicó el test de Tukey. Finalmente, una prueba post hoc sobre los residuos y estadísticos fue realizado para confirmar los resultados de la hipótesis. 


\section{Resultados}

\section{Hidrogramas y volumen de descarga de agua superficial en la salida del reguero}

En la Figura $\mathrm{N}^{\circ}$ 3, fueron representadas los valores de caudal instantáneo del flujo (Fig. 3a) y el volumen acumulado de la descarga de agua al final de la cárcava durante los experimentos (Fig. 3b). Como complemento, en el Cuadro 2 fueron añadidos también los principales estadísticos descriptivos y los umbrales de significancia para destacar si existían los resultados de cada repetición.

\section{Cuadro 2.}

Estadísticas descriptivas de la velocidad y el incremento de la descarga total por intervalo de agua a lo largo de la cárcava

\begin{tabular}{|c|c|c|c|c|c|c|}
\hline Variables & \multicolumn{2}{|c|}{ Velocidad $\left(\mathrm{m} \mathrm{s}^{-1}\right)$} & \multicolumn{2}{|c|}{ Descarga $\left(\mid \mathrm{s}^{-1}\right)$} & \multicolumn{2}{|c|}{$\begin{array}{c}\text { Volumen de descarga } \\
\text { acumulado (I) }\end{array}$} \\
\hline Repeticiones & A & $B$ & A & $B$ & $A$ & $\mathrm{~B}$ \\
\hline Promedio & 27,6 & 29,8 & 0,92 & 1,03 & 634,0 & 804,0 \\
\hline Desviación estándar & 16,5 & 22,9 & 0,55 & 0,76 & - & . \\
\hline Máximos absolutos & 49,5 & 60,7 & 1,6 & 2,0 & - & . \\
\hline Diferencias* & \multicolumn{2}{|r|}{$p<0,704$} & \multicolumn{2}{|r|}{$p<0,040$} & & . \\
\hline
\end{tabular}

A: Repetición bajo condiciones de aridez; B: Repetición bajo condiciones de humedad. *Test de Tukey.

Figura N.० 3.

Descarga de agua durante la simulación (a) y volumen total acumulado (b)

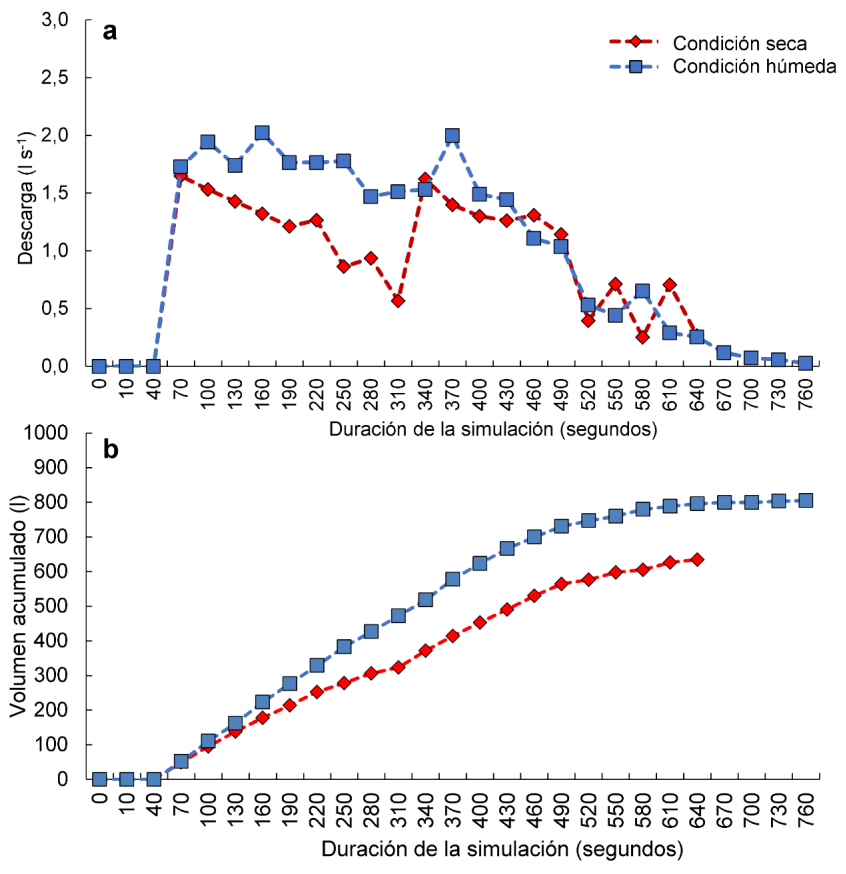


En primer lugar, en relación al volumen del flujo, se puede observar como en la segunda repetición bajo condiciones de suelo húmedo (segunda simulación), el volumen en casi todos los momentos del experimento supera a la de la primera. Para el hidrograma B (condición húmeda), se alcanzaron promedios de 1,0 I s-1, por 0,92 I s-1 del hidrograma A (condición seca). También los caudales punta fueron más elevados en la segunda repetición (condiciones húmedas), registrando valores de 2,0 I s-1 $1,6 \mid \mathrm{s} \mathrm{s}^{-1}$, respectivamente. Sin embargo, tras aplicar el test de Tukey, dichas diferencias no pueden ser constatadas como significativas estadísticamente $(p<0,704)$. Por lo tanto, solo se pueden confirmar ligeras diferencias en cuanto a este parámetro a lo largo del experimento y breves cambios en cortos tramos al inicio.

Por otro lado, en relación a la descarga total de agua, en la primera repetición un volumen de 634 I, lo que equivale a un $63,4 \%$ de la cantidad de agua bombeada, fue expulsada por la boca de la cárcava. Al terminar la segunda simulación, un total de 805 litros fueron desaguados por la cárcava (80,5\%). Esta diferencia de valores provocó que el test de Tukey remarcara que, en este parámetro hidráulico, sí existen diferencias significativamente estadísticas $(p<0,04)$.

\section{Velocidad del frente y del flujo durante la simulación de escorrentía mediante el uso de trazadores}

A continuación, se procedió al análisis de la velocidad del frente de la escorrentía con el uso de trazadores a lo largo de cada simulación. Dichos resultados fueron representados en el cuadro 3 y la Figura $n^{\circ} 4$ junto con el perfil longitudinal de la cárcava, con objeto de relacionar cada fluctuación con algún cambio en la inclinación. Además, también se incluyeron estadísticos descriptivos para apoyar la evaluación de los resultados y un análisis de las diferencias entre repeticiones.

\section{Cuadro 3.}

Estadísticas descriptivas de la velocidad del frente inicial de escorrentía y durante la simulación con el uso de trazadores.

\begin{tabular}{|c|c|c|c|c|c|c|}
\hline Variables & \multicolumn{2}{|c|}{ Frentes } & \multicolumn{2}{|c|}{ Trazador 1} & \multicolumn{2}{|c|}{ Trazador 2} \\
\hline Repeticiones & A & B & A & B & A & B \\
\hline Promedio $\left(\mathrm{m} \mathrm{s}^{-1}\right)$ & 0,46 & 0,40 & 2 & 2,8 & 2 & 2,2 \\
\hline Desviación estándar $\left(\mathrm{m} \mathrm{s}^{-1}\right)$ & 1,6 & 0,7 & 0,8 & 1,1 & 0,8 & 0,8 \\
\hline Máximos absolutos $\left(\mathrm{m} \mathrm{s}^{-1}\right)$ & 6,7 & 3,3 & 3,4 & 4,4 & 3,5 & 3,3 \\
\hline Mínimos absolutos $\left(\mathrm{m} \mathrm{s}^{-1}\right)$ & 1,1 & 1 & 0,8 & 0,3 & 0,5 & 0,6 \\
\hline Diferencias* & \multicolumn{2}{|r|}{$p<0,319$} & \multicolumn{2}{|r|}{$p<0,164$} & \multicolumn{2}{|r|}{$p<0,460$} \\
\hline
\end{tabular}

A: Repetición bajo condiciones de aridez; B: Repetición bajo condiciones de humedad. *: Test de Tukey. 
Figura N. ${ }^{\circ} 4$.

Velocidad del frente del flujo de agua y perfil longitudinal de la cárcava.

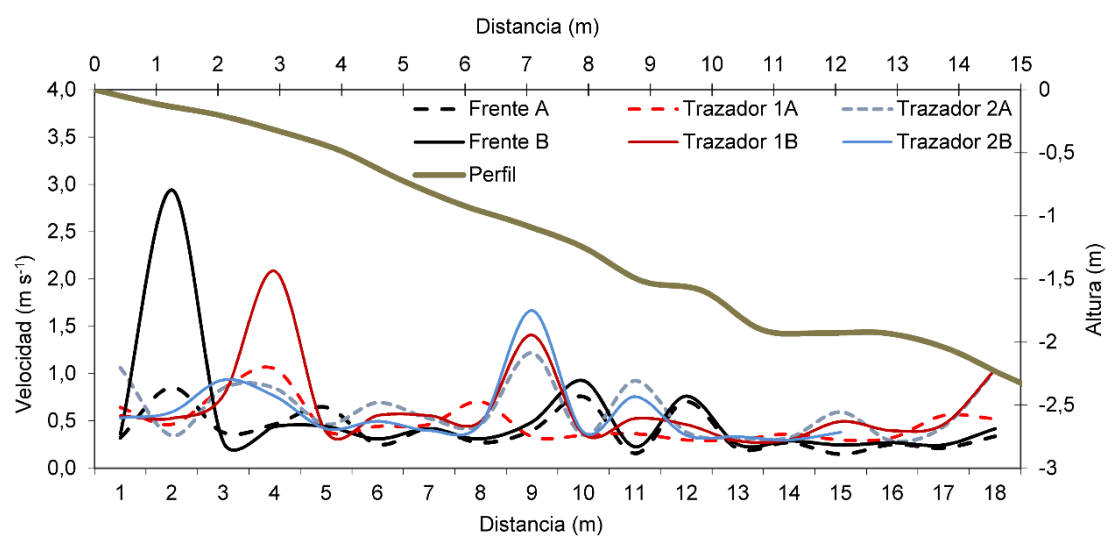

El perfil longitudinal de la cárcava muestra una elevación de la pendiente desde el intervalo 0-1 m hasta 5-6 m, pasando desde 5 a 13,30. A partir de ahí, el perfil mostró una serie de fluctuaciones, donde se aprecian máximas inclinaciones de 15,70 en los intervalos 9-10 hasta zonas totalmente planas entre el 12 y $13 \mathrm{~m}$. Solo fueron localizados tres nickpoints, siendo los más relevantes los que se localizan entre los 10 y 11 metros con unos $12^{\circ}$, y en el tramo donde comienza la zona más llana, con una inclinación de $22,7^{\circ}$.

Respecto a los frentes iniciales A y B, solo se aprecian diferencias de velocidad en los tres primeros metros de la cárcava, pero en general, cabe resaltar que la velocidad es mayor en la curva $B\left(0,54 \mathrm{~m} \mathrm{~s}^{-1}\right)$ que en la $A\left(0,39 \mathrm{~m} \mathrm{~s}^{-1}\right)$. El frente $A$ alcanza los mayores valores al inicio de la cárcava y del experimento, alcanzando los $0,85 \mathrm{~m} \mathrm{~s}^{-1}$ hasta los tres primeros metros. Entre los metros $3 \mathrm{y}$ 10 metros, se puede apreciar cómo se mantienen unos niveles similares y sin picos. Sin embargo, en las zonas donde la pendiente aumenta se reconocen de nuevo ligeros incrementos. Finalmente, cabe destacar como en la zona plana se registra un descenso de la velocidad, alcanzando en ambos frentes valores de $1 \mathrm{~m} \cdot \mathrm{s}^{-1}$.

Con respecto a los trazadores utilizados, se observaron similares patrones de velocidad que en los frentes iniciales de flujo A y $B$ descritos anteriormente. Sin embargo, la mayor diferencia es que durante el experimento $B$, la velocidad de ambos trazadores aumentó $\left(0,49\right.$ vs $0,54 \mathrm{~m} \mathrm{~s}^{-1} ; 0,62$ vs $0,65 \mathrm{~m} \mathrm{~s}^{-1}$ ). Los picos de flujo se registraron justo en los intervalos siguientes a los registrados en los frentes A y B y antes de alcanzar el salto de pendiente a partir de los 9 metros.

Pese a que el test de Tukey no permite confirmar diferencias significativamente estadísticas, entre las curvas bajo condiciones secas y húmedas, a simple vista el frente $A$, al inicio, muestra mayores velocidades que los trazadores utilizados durante la segunda repetición, indicando que, bajo condiciones de humedad previa, la velocidad del flujo es menor al comienzo de la cárcava, pero aumenta cerca de la boca mostrando picos más elevados y constantes. 


\section{Concentración de sedimentos y cambios morfológicos en el perfil transversal de la cárcava}

En la Figura N. ${ }^{\circ}$ 5, es posible observar las diferencias de concentración de sedimentos que la escorrentía es capaz de transportar en diferentes puntos de la cárcava y durante distintos tiempos de medida bajo condiciones secas (A) y humedas (B).

Figura N. ${ }^{0} 5$.

Concentración de sedimentos bajo condiciones de aridez (A) y humedad (B).
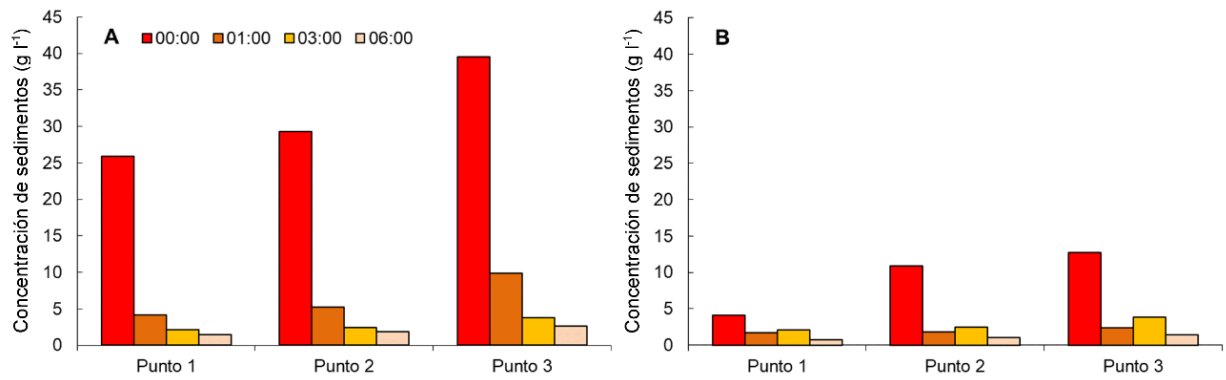

En la primera repetición $(A)$, la concentración de sedimentos instantánea presentó valores superiores a los de la segunda (B) en los tres puntos de análisis con medias de aproximadamente $11 \mathrm{~g} \mathrm{l}^{-1}$ y $4 \mathrm{~g} \mathrm{l}^{\left.\right|^{-1}}$ respectivamente, como resultado de los procesos de lavado del material suelto y/o depositado en la cárcava que caracterizó el experimento en condiciones secas. Es significativo como en la primera repetición en el punto 3 se llegan alcanzar al inicio del hidrograma concentraciones de hasta $40 \mathrm{~g} \mathrm{l}^{-1}$ que se van reduciendo a medida que se incrementa el lavado. En el experimento bajo condiciones húmedas, a pesar de los caudales superiores, los valores de concentración fueron notablemente más bajos con un valor máximo de $13 \mathrm{~g} \mathrm{l}^{-1} \mathrm{en}$ el mismo punto de muestro y en el mismo instante. También se destaca la alta correlación de los valores de concentración instantánea en los dos experimentos $(r=0,88, p>0,05)$ a pesar de sus diferencias en magnitud, donde se pone en evidencia el impacto de un mismo evento bajo condiciones de disponibilidad de sedimentos sueltos diferentes (antes y después del lavado). Finalmente, son significativas las diferencias de arrastre de sedimentos, si se aproximan los valores de concentración de sedimentos del punto 3 a los hidrogramas tomados en la salida, con descargas de sedimentos de casi $3,0 \mathrm{~kg}$ para la repetición A frente a $0,9 \mathrm{~kg}$ de la B, a pesar de mayores volúmenes de flujo.

Finalmente, en la Figura N. ${ }^{\circ}$ 6, fueron representados antes de comenzar la simulación, los cambios en el perfil transversal de la cárcava en los tres puntos de muestreo, tras concluir el primer experimento y tras finalizar la segunda repetición. De esta forma, es posible observar que la escorrentía ha provocado un promedio de rebajes de hasta $9 \mathrm{~cm}$, con máximos de 13 y mínimos de 2. En todos los puntos, se han generado los mismos promedios de rebajes, demostrando la elevada erodibilidad del suelo en todos los puntos de medición. 
Figura N. ${ }^{\circ} 6$.

Secciones transversales de la cárcava a diferentes longitudes.

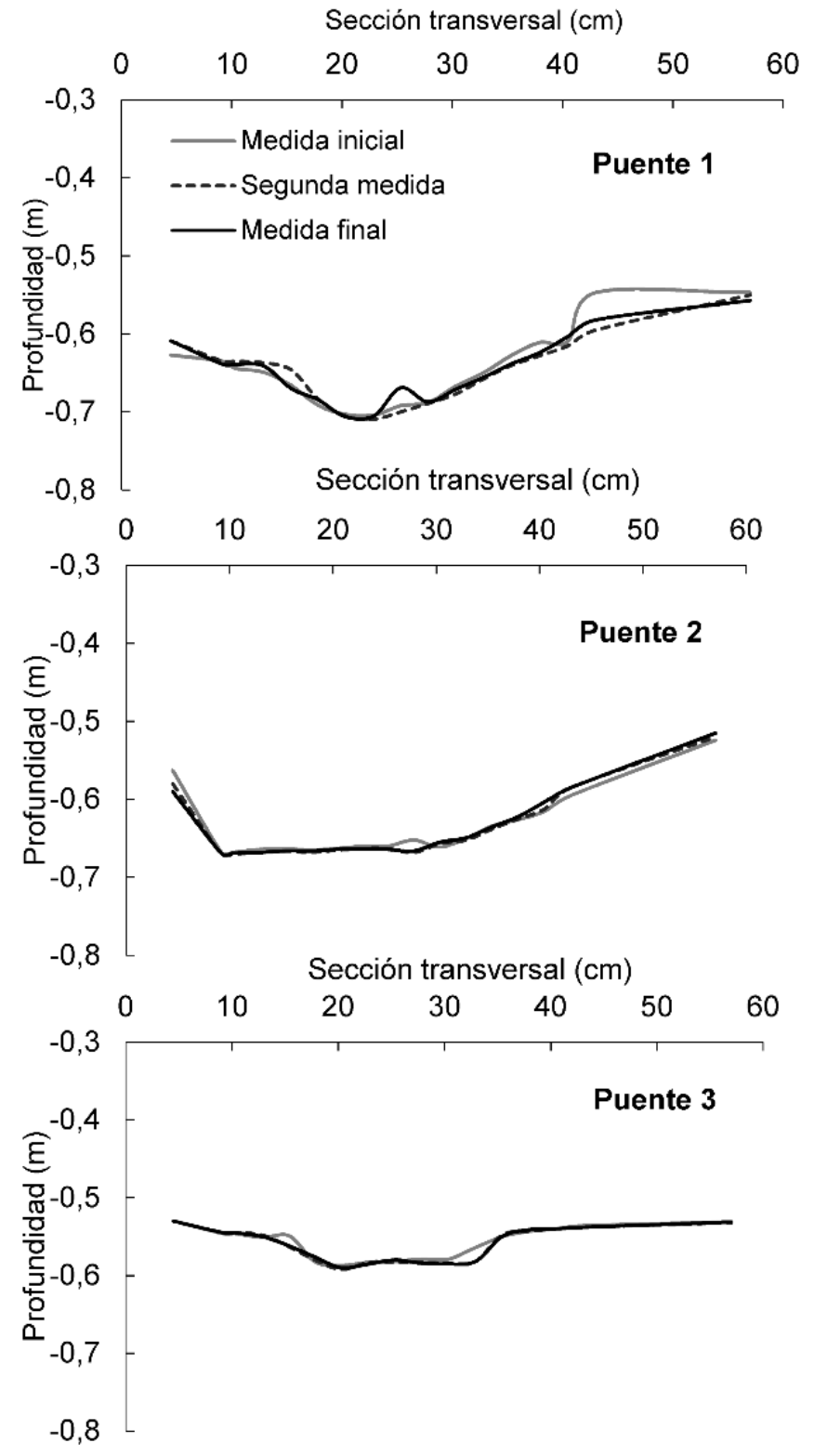

\section{Discusión}

La erosión del suelo en campos cultivados es un problema de interés global por su impacto negativo en los ecosistemas naturales o en el calentamiento global (García-Ruiz et al. 2015; Nearing et al. 2017) Sin embargo, todavía hay mucho que hacer en términos de medidas de mejora de conservación de suelos y planificación territorial (Lasanta et al.2015; Martínez-Casasnovas et al. 2010) 
En los campos cultivados con olivar, numerosos autores han demostrado que las tasas de erosión son muy elevadas por la interacción de diversos factores como la pendiente, el manejo y la alta erosividad de eventos periódicos, donde además la mayoría de esfuerzos se han dedicado a reducir la erosión de sedimentos derivada de procesos de ladera donde el uso de cubiertas vegetales o inertes ha demostrado sus beneficios (Gómez et al. 2014). Dentro de las formas de erosión más comunes en las áreas de cultivo, encontramos las cárcavas, siendo pocos los estudios que se centran en cuantificar a escala pedón, las pérdidas de agua y suelo en tiempo real bajo condiciones extremas. Dicha laguna de información se debe posiblemente a la elevada variación intra- e inter-anual de las precipitaciones y al reducido número de eventos erosivos extremos registrados en las áreas mediterráneas, lo cual dificulta las mediciones in situ y su planificación para estudios con duración de 2 a 4 años (Martínez-Casasnovas et al. 2002 ; Raveh-Rubin y Wernli 2016). Sin embargo, el uso de mediciones experimentales en el campo como las simulaciones de lluvia, viento o escorrentía, facilitan esta tarea exponencialmente (Marzen et al. 2017).

Tal y como ha demostrado el presente estudio y otros previos (e.g. Castillo y Gómez, 2016; Taguas et al., es fundamental prestar atención a la erosión derivada del flujo concentrado de regueros y cárcavas donde eventos frecuentes (de periodos de retorno de 1 año o incluso menores) pueden contribuir significativamente a la exportación de agua y sedimentos en cuencas incluso bajo condiciones de muy baja humedad del suelo. Aunque todavía hay poca información al respecto, el uso de las simulaciones de escorrentía permite cuantificar el efecto negativo de cárcavas originadas por lluvias previas en el suelo y hacerlas comparables con otras áreas de estudio para ponerlas en contexto (Wirtz et al., 2010; 2012; 2013). En este sentido, ampliar el rango de condiciones es más que deseable, especialmente porque además permitiría implementar y/o calibrar algoritmos dedicados a la simulación de regueros y cárcavas tal puede ser el caso de SWAT (Cibin et al., 2010) Sin embargo, Wirtz et al., (2012) aplicó WEPP.

Con toda lógica, sería interesante repetir este experimento en áreas con mayores pendientes (Nadal-Romero et al. 2014), en cárcavas generadas por las rodadas de tractores (Arnáez et al., 2012), en suelos con cobertura vegetal y con diferente sustrato litológico (Asbjornsen et al. 2014; Orgill et al. 2017). Sin embargo, debido al elevado coste de tiempo (un día para dos experimentos), transporte de materiales (1000 litros de agua, trazadores, sensores, etc.) y de personal (más de 10 personas), el número de repeticiones puede resultar ciertamente limitante.

A continuación se presentan algunos ejemplos extraídos de los trabajo de Wirtz et al., (2012; 2013) para comparar los valores de concentración instantánea de sedimentos bajo distintos suso de suelo por ejemplo, en Arnás (Pirineo Central, Aragón, España) en áreas dedicadas al pasto sobre areniscas con material carbonatado y estratos margosos, se registraron concentración de sedimentos de hasta 6,3 $\mathrm{g} \mathrm{l}^{-1}$ y velocidades de 0,13 $\mathrm{m} \mathrm{s}^{-1}$. En Belerda (Granada, España), en campos cultivados con almendros y áreas abandonadas sobre materiales terciarios y conglomerados cuaternarios, se obtuvieron hasta $54,3 \mathrm{~g} \mathrm{l}^{-1} \mathrm{y}$ velocidades de $0,5 \mathrm{~m} \mathrm{~s}^{-1}$. De esta forma, se observa como en el olivar, los picos máximos de concentración de sedimentos alcanzaron valores similares a los usos de suelo con especies leñosas $\left(40 \mathrm{~g} \mathrm{l}^{-1}\right)$. No obstante, la velocidad promedio es mucho más elevada, incluso que en cárcavas de origen antrópico (agri-spillways) excavadas por los viticultores de los Montes de Málaga para desaguar la escorrentía sobrante (Rodrigo-Comino et al. 2017). Por lo tanto, se podría confirmar que además de un problema con la erosión del suelo, también es relevante el problema con la pérdida de agua. Como numerosos autores destacaron previamen- 
te, elevadas tasas de escorrentía pueden, además de transportar gran cantidad de sedimentos e incrementar el tamaño de las cárcavas (Bertalan et al. 2016), también pueden transportar una gran carga de nutrientes, productos químicos y restos de vegetación, contaminando los cauces ladera abajo y provocando fuertes crecidas del caudal de forma esporádica (Angrill et al. 2017; López-Vicente et al. 2016). Además de la conservación de la calidad del agua, medidas de control deberían ser encauzadas a redirigir el agua sobrante a embalses o balsas para regadío y así reducir los problemas causados por la aridez durante los meses de verano que acusan los climas mediterráneos (Ruiz-Sinoga et al. 2012).

Nuestros resultados también permiten comparar las dinámicas erosivas con otros estudios realizados en la misma cuenca experimental con otros métodos. Taguas et al., (2013) a escala cuenca utilizando un amplio rango de datos (1946-2005) y un análisis de componentes principales, confirmó que las mayores tasas de erosión en un período de retorno de 3 años, fueron provocadas por pocos eventos lluviosos de alta erosividad. Dichos eventos causaron tasas de 7,2 a $17 \mathrm{t}$ de pérdida de suelo, gran parte, canalizadas a través de las cárcavas y regueros de la cuenca. También Taguas et al., (2015) destacaron que el número de cárcavas y regueros está en avance en la cuenca, asociados con eventos de precipitación de 30 minutos con períodos de retorno superiores a 2 años. Esto reafirma nuestros resultados y nuestra petición de informar a las autoridades de la necesidad de implantar medidas para frenar el crecimiento de las cárcavas. Como nuestro estudio confirmó, gran parte de los eventos frecuentes que ocurren en el área de estudio, tienen rápidas respuestas erosivas. Las soluciones posibles, podrían pasar por inclusión del uso de cobertura vegetal (Ruiz-Colmenero et al. 2013) para procesos de ladera y erosión laminar. También serían pertinentes las actuaciones en forma de pequeñas presas o diques de retención para reducir las velocidades y corregir las pendientes. Esta medida sería complementaria a la revegetación de los cauces con especies autóctonas que actúen de buffer o al menos reducir las áreas de labor particularmente en grandes cárcavas, respetando los cauces para que la vegetación espontáneamente estabilice los taludes.

\section{Conclusiones}

A través de la simulación de escorrentía, se ha demostrado como cárcavas generadas en campos cultivados con olivar convencional son capaces de canalizar elevadas cantidades de suelo y agua. Bajo condiciones de aridez y humedad, a través del bombeo de 1000 litros de agua, más del 60 y $80 \%$ ha sido canalizado y expulsado por la boca de la cárcava. Los resultados del cálculo de la concentración de sedimentos han registrado un incremento desde el principio de la cárcava hasta su final por motivos de la dinámica de arrastre, alcanzando valores de hasta $40 \mathrm{~g} / \mathrm{l}$. Dicha erosión también se manifestó en los cambios de hasta $9 \mathrm{~cm}$ en el perfil transversal de la cárcava, demostrando que la escorrentía generó una clara incisión en el suelo, eliminando parte del horizonte fértil. Por lo tanto, queremos llamar la atención de los agricultores y planificadores del territorio con objeto de insistir en la implantación de medidas que permitan controlar la erosión derivadas del flujo concentrado. Entre dichas medidas, la reducción de las cárcavas y el uso de una cobertura vegetal localizada en las áreas de generación de escorrentía son vitales para no perder suelo, nutrientes, y agua. 


\section{Agradecimientos}

Nos gustaría agradecer a los agricultores Antonio Muñoz and Esteban Ruiz por facilitarnos el acceso a las áreas de estudio. También, es importante agradecer la labor de Francisco Montero, trabajador de la empresa pública de gestión EGEMASA y al Ayuntamiento de Puente Genil por todas las facilidades para gestionar la campaña de campo. También queremos agradecer a los estudiantes de grado y master de las Universidades de Córdoba y Tréveris por su duro trabajo en el campo y en el laboratorio, su buena disposición y alegría. Esta investigación fue apoyada por el proyecto de investigación CGL2015-64284-C2-2-R, financiado por el MINECO-FEDER (Ministerio de Economía y Competitividad) y la Acción Cost (Connecteur) adjudicada al Dr. Manuel Seeger.

\section{Bibliografía}

ALBARADEYIA, I., HANI, A. y SHAHROUR, I., 2011. WEPP and ANN models for simulating soil loss and runoff in a semi-arid Mediterranean region. Environmental Monitoring and Assessment, vol. 180, no. 1-4, pp. 537-556. DOI 10.1007/s10661-010-1804-x.

ALONSO, C.V., BENNETT, S.J. y STEIN, O.R., 2002. Predicting head cut erosion and migration in concentrated flows typical of upland areas. Water Resources Research, vol. 38, no. 12, pp. 39-139-15. DOI 10.1029/2001WRO01173.

ÁLVAREZ-MOZOS, J., ABAD, E., GIMÉNEZ, R., CAMPO, M.A., GOÑI, M., ARIVE, M., CASALÍ, J., DíEZ, J. y DIEGO, I., 2014. Evaluation of erosion control geotextiles on steep slopes. Part 1: Effects on runoff and soil loss. CATENA, vol. 118, no. Supplement C, pp. 168-178. DOI 10.1016/j.catena.2013.05.018.

ANGRILL, S., PETIT-BOIX, A., MORALES-PINZÓN, T., JOSA, A., RIERADEVALL, J. y GABARRELL, X., 2017. Urban rainwater runoff quantity and quality - A potential endogenous resource in cities? Journal of Environmental Management, vol. 189, pp. 14-21. DOI 10.1016/j.jenvman.2016.12.027.

ARNÁEZ, J., RUIZ, P., LASANTA, T., ORTIGOSA, L.M., LLORENTE, J.A., PASCUAL, N.E. y LANA-RENAULT, N., 2012. Efectos de las rodadas de tractores en la escorrentía y erosión de suelos en laderas cultivadas con viñedos. Cuadernos de investigación geográfica, no. 38, pp. 115-130.

ASBJORNSEN, H., HERNANDEZ-SANTANA, V., LIEBMAN, M., BAYALA, J., CHEN, J., HELMERS, M., ONG, C.K. y SCHULTE, L.A., 2014. Targeting perennial vegetation in agricultural landscapes for enhancing ecosystem services. Renewable Agriculture and Food Systems, vol. 29, no. 2, pp. 101-125. 1742-1713. DOI 10.1017/S1742170512000385.

BEN SLIMANE, A., RACLOT, D., EVRARD, O., SANAA, M., LEFEVRE, I. y LE BISSONNAIS, Y., 2015. Relative contribution of rill/interrill and gully/channel erosion to small reservoir siltation in Mediterranean environments. Land Degradation \& Development, vol. 27, no. 3, pp. 785-797. DOI 10.1002/ Idr.2387.

BERTALAN, L., TÓTH, C.A., SZABÓ, G., NAGY, G., KUDA, F. y SZABÓ, S., 2016. Confirmation of a theory: reconstruction of an alluvial plain development in a flume experiment. Erdkunde, vol. 70, no. 3, pp. 271-285. DOI 10.3112/erdkunde.2016.03.05. 
BINGNER, R., THEURER, F.D. y YUAN, Y., 2011. Technical Processes Documentation Version 5.2. MS, USA: USDA-ARS, National Sedimentation Laboratory, Oxford.

BURGUET, M., TAGUAS, E.V., CERDÀ, A. y GÓMEZ, J.A., 2016. Soil water repellency assessment in olive groves in Southern and Eastern Spain. CATENA, vol. 147, pp. 187-195. DOI 10.1016/j.catena.2016.07.005.

CASTILLO, C. y GÓMEZ, J.A., 2016. A century of gully erosion research: Urgency, complexity and study approaches. Earth-Science Reviews, vol. 160, pp. 300-319. DOI 10.1016/j.earscirev.2016.07.009.

CHOW, V.T., MAIDMENT, D.R. y MAYS, L.W., 1994. Hidrología aplicada. S.I.: McGraw-Hill International editions.

CIBIN R., SUDHEER K. P. y CHAUBEY I., 2010. Sensitivity and identifiability of stream flow generation parameters of the SWAT model. Hydrological Processes, vol. 24, no. 9, pp. 1133-1148. DOI 10.1002/hyp.7568.

CONFORTI, M., BUTTAFUOCO, G., LEONE, A.P., AUCELLI, P.P.C., ROBUSTELLI, G. y SCARCIGLIA, F., 2013. Studying the relationship between water-induced soil erosion and soil organic matter using Vis-NIR spectroscopy and geomorphological analysis: A case study in southern Italy. CATENA, vol. 110, pp. 44-58. DOI 10.1016/j.catena.2013.06.013.

DI STEFANO, C., FERRO, V., BURGUET, M. y TAGUAS, E.V., 2016. Testing the long term applicability of USLE-M equation at a olive orchard microcatchment in Spain. CATENA, vol. 147, pp. 71-79. DOI 10.1016/j.catena.2016.07.001.

FLESKENS, L. y STROOSNIJDER, L., 2007. Is soil erosion in olive groves as bad as often claimed? Geoderma, vol. 141, no. 3-4, pp. 260-271. DOI 10.1016/j.geoderma.2007.06.009.

FRANCO MARTÍNEZ, J.A. y RODRÍGUEZ ENTRENA, M., 2009. Adopción y difusión de la agricultura ecológica en España. Factores de reconversión en el olivar andaluz. Cuadernos de Economía, vol. 32, no. 90, pp. 137-158. DOI 10.1016/S0210-0266(09)70055-X.

GARCÍA-DÍAZ, A., BIENES, R., SASTRE, B., NOVARA, A., GRISTINA, L. y CERDÀ, A., 2017. Nitrogen losses in vineyards under different types of soil groundcover. A field runoff simulator approach in central Spain. Agriculture, Ecosystems \& Environment, vol. 236, pp. 256-267. DOI 10.1016/j. agee.2016.12.013.

GARCÍA-DÍAZ, A., MARQUÉS, M.J., SASTRE, B. y BIENES, R., 2017. Labile and stable soil organic carbon and physical improvements using groundcovers in vineyards from central Spain. The Science of the Total Environment, vol. 621, pp. 387-397. DOI 10.1016/j.scitotenv.2017.11.240.

GARCÍA-RUIZ, J.M., BEGUERÍA, S., NADAL-ROMERO, E., GONZÁLEZ-HIDALGO, J.C., LANA-RENAULT, N. y SANJUÁN, Y., 2015. A meta-analysis of soil erosion rates across the world. Geomorphology, vol. 239, pp. 160-173. DOI 10.1016/j.geomorph.2015.03.008. 
GARCÍA-RUIZ, J.M., NADAL-ROMERO, E., LANA-RENAULT, N. y BEGUERÍA, S., 2013. Erosion in Mediterranean landscapes: Changes and future challenges. Geomorphology, vol. 198, pp. 20-36. DOI 10.1016/j.geomorph.2013.05.023.

GÓMEZ, J.A., GIRÁLDEZ, J.V., PASTOR, M. y FERERES, E., 1999. Effects of tillage method on soil physical properties, infiltration and yield in an olive orchard. Soil and Tillage Research, vol. 52, no. 3-4, pp. 167-175. DOI 10.1016/S0167-1987(99)00078-1.

GÓMEZ, J.A., GIRÁLDEZ, J.V. y VANWALLEGHEM, T., 2008. Comments on "Is soil erosion in olive groves as bad as often claimed?" by L. Fleskens and L. Stroosnijder. Geoderma, vol. 147, no. 1-2, pp. 93-95. DOI 10.1016/j.geoderma.2008.07.006.

GÓMEZ, J.A., GUZMÁN, M.G., GIRÁLDEZ, J.V. y FERERES, E., 2009. The influence of cover crops and tillage on water and sediment yield, and on nutrient, and organic matter losses in an olive orchard on a sandy loam soil. Soil and Tillage Research, vol. 106, no. 1, pp. 137-144. DOI 10.1016/j. still.2009.04.008.

GÓMEZ, J.A., INFANTE-AMATE, J., DE MOLINA, M.G., VANWALLEGHEM, T., TAGUAS, E.V. y LORITE, I., 2014. Olive cultivation, its impact on soil erosion and its progression into yield impacts in Southern Spain in the past as a key to a future of increasing climate uncertainty. Agriculture, vol. 4, no. 2, pp. 170-198. DOI 10.3390/agriculture4020170.

HUESO-GONZÁLEZ, P., MARTíNEZ-MURILLO, J.F. y RUIZ-SINOGA, J.D., 2014. The impact of organic amendments on forest soil properties under Mediterranean climatic conditions. Land Degradation \& Development, vol. 25, no. 6, pp. 604-612. DOI 10.1002/ldr.2296.

KAIRIS, O., KARAVITIS, C., KOUNALAKI, A., SALVATI, L. y KOSMAS, C., 2013. The effect of land management practices on soil erosion and land desertification in an olive grove. Soil Use and Management, vol. 29, no. 4, pp. 597-606. DOI 10.1111/sum.12074.

KAVIAN, A., GOLSHAN, M. y ABDOLLAHI, Z., 2017. Flow discharge simulation based on land use change predictions. Environmental Earth Sciences, vol. 76, no. 16, pp. 588. DOI 10.1007/s12665017-6906-0.

KAVIAN, A., MOHAMMADI, M., GHOLAMI, L. y RODRIGO-COMINO, J., 2018. Assessment of the spatiotemporal effects of land use changes on runoff and nitrate Loads in the Talar River. Water, vol. 10, no. 4, pp. 445. DOI 10.3390/w10040445.

KRAUSHAAR, S., HERRMANN, N., OLLESCH, G., VOGEL, H.-J. y SIEBERT, C., 2014. Mound measurements - quantifying medium-term soil erosion under olive trees in Northern Jordan. Geomorphology, vol. 213, pp. 1-12. DOI 10.1016/j.geomorph.2013.12.021.

LASANTA, T., NADAL-ROMERO, E. y ARNÁEZ, J., 2015. Managing abandoned farmland to control the impact of re-vegetation on the environment. The state of the art in Europe. Environmental Science \& Policy, vol. 52, pp. 99-109. DOI 10.1016/j.envsci.2015.05.012. 
LÓPEZ-VICENTE, M., NADAL-ROMERO, E. y CAMMERAAT, E.L.H., 2016. Hydrological connectivity does change over 70 years of abandonment and afforestation in the Spanish Pyrenees. Land Degradation \& Development, vol. 28 (4), 1298-1310. DOI 10.1002/ldr.2531.

MARTÍNEZ-CASASNOVAS, J.A., RAMOS, M.C. y BALASCH, C., 2013. Precision analysis of the effect of ephemeral gully erosion on vine vigour using NDVI images. En: J.V. STAFFORD (ed.), Precision agriculture '13. Wageningen: Wageningen Academic Publishers, pp. 777-783. [Consulta: 26 julio 2016]. ISBN 978-90-8686-778-3.

MARTÍNEZ-CASASNOVAS, J.A., RAMOS, M.C. y COTS-FOLCH, R., 2010. Influence of the EU CAP on terrain morphology and vineyard cultivation in the Priorat region of NE Spain. Land Use Policy, vol. 27, no. 1, pp. 11-21. DOI 10.1016/j.landusepol.2008.01.009.

MARTÍNEZ-CASASNOVAS, J.A., RAMOS, M.C. y RIBES-DASI, M., 2002. Soil erosion caused by extreme rainfall events: mapping and quantification in agricultural plots from very detailed digital elevation models. Geoderma, vol. 105, no. 1-2, pp. 125-140. DOI 10.1016/S0016-7061(01)00096-9.

MARTÍNEZ-CASASNOVAS, J.A. y SÁNCHEZ-BOSCH, I., 2000. Impact assessment of changes in land use/conservation practices on soil erosion in the Penedès-Anoia vineyard region (NE Spain). Soil and Tillage Research, vol. 57, no. 1-2, pp. 101-106. DOI 10.1016/S0167-1987(00)00142-2.

MARZEN, M., ISERLOH, T., DE LIMA, J.L.M.P., FISTER, W. y RIES, J.B., 2017. Impact of severe rain storms on soil erosion: Experimental evaluation of wind-driven rain and its implications for natural hazard management. Science of The Total Environment, vol. 590-591, pp. 502-513. DOI 10.1016/j. scitotenv.2017.02.190.

MARZOLFF, I. y POESEN, J., 2009. The potential of 3D gully monitoring with GIS using high-resolution aerial photography and a digital photogrammetry system. Geomorphology, vol. 111, no. 1-2, pp. 48-60. DOI 10.1016/j.geomorph.2008.05.047.

NADAL-ROMERO, E., PETRLIC, K., VERACHTERT, E., BOCHET, E. y POESEN, J., 2014. Effects of slope angle and aspect on plant cover and species richness in a humid Mediterranean badland. Earth Surface Processes and Landforms, vol. 39, no. 13, pp. 1705-1716. DOI 10.1002/esp.3549.

NEARING, M.A., POLYAKOV, V.O., NICHOLS, M.H., HERNANDEZ, M., LI, L., ZHAO, Y. y ARMENDARIZ, G., 2017. Slope-velocity equilibrium and evolution of surface roughness on a stony hillslope. $\mathrm{Hy}$ drology and Earth System Sciences, vol. 21, no. 6, pp. 3221-3229. DOI 10.5194/hess-21-3221-2017.

ORGILL, S.E., CONDON, J.R., CONYERS, M.K., MORRIS, S.G., MURPHY, B.W. y GREENE, R.S.B., 2017. Parent material and climate affect soil organic carbon fractions under pastures in south-eastern Australia. Soil Research, vol. 55 (8) DOI 10.1071/SR16305.

PARRAS-ALCÁNTARA, L., LOZANO-GARCÍA, B., KEESSTRA, S., CERDÀ, A. y BREVIK, E.C., 2016. Longterm effects of soil management on ecosystem services and soil loss estimation in olive grove top soils. Science of The Total Environment, vol. 571, pp. 498-506. DOI 10.1016/j.scitotenv.2016.07.016. 
RAVEH-RUBIN, S. y WERNLI, H., 2016. Large-scale wind and precipitation extremes in the Mediterranean: dynamical aspects of five selected cyclone events. Quarterly Journal of the Royal Meteorological Society, vol. 142, no. 701, pp. 3097-3114. DOI 10.1002/qj.2891.

RODRIGO-COMINO, J., GIMÉNEZ-MORERA, A., PANAGOS, P., POURGHASEMI, H.R., PULIDO, M. y CERDÀ, A., 2019. The potential of straw mulch as a nature-based solution for soil erosion in olive plantation treated with glyphosate: A biophysical and socioeconomic assessment. Land Degradation \& Development, en prensa. DOI 10.1002/ldr.3305.

RODRIGO-COMINO, J., TAGUAS, E., SEEGER, M. y RIES, J.B., 2018. Quantification of soil and water losses in an extensive olive orchard catchment in Southern Spain. Journal of Hydrology, vol. 556, pp. 749-758. DOI 10.1016/j.jhydrol.2017.12.014.

RODRIGO-COMINO, J., WIRTZ, S., BREVIK, E.C., RUIZ-SINOGA, J.D. y RIES, J.B., 2017. Assessment of agri-spillways as a soil erosion protection measure in Mediterranean sloping vineyards. Journal of Mountain Science, vol. 14, no. 6, pp. 1009-1022. DOI DOI: 10.1007/s11629-016-4269-8.

RUIZ-COLMENERO, M., BIENES, R., ELDRIDGE, D.J. y MARQUES, M.J., 2013. Vegetation cover reduces erosion and enhances soil organic carbon in a vineyard in the central Spain. Catena, vol. 104, pp. 153-160. DOI 10.1016/j.catena.2012.11.007.

RUIZ-SINOGA, J.D., GARCIA-MARIN, R., GABARRON-GALEOTE, M.A. y MARTINEZ-MURILLO, J.F., 2012. Analysis of dry periods along a pluviometric gradient in Mediterranean southern Spain. International Journal of Climatology, vol. 32, no. 10, pp. 1558-1571. DOI 10.1002/joc.2376.

SMITH, P., 2018. Managing the global land resource. Proc. R. Soc. B, vol. 285, no. 1874, pp. $1471-$ 2954. DOI 10.1098/rspb.2017.2798.

TAGUAS, Encarnación, YUAN, Y., PEÑA, A. y AYUSO, J.L., 2010. Predicción de cárcavas efímeras mediante el índice topográfico combinado en una microcuenca de olivar en Andalucía (España). Agrociencia, vol. 44, no. 4, pp. 409-426.

TAGUAS, E.V., AYUSO, J.L., PÉREZ, R., GIRÁLDEZ, J.V. y GÓMEZ, J.A., 2013. Intra and inter-annual variability of runoff and sediment yield of an olive micro-catchment with soil protection by natural ground cover in Southern Spain. Geoderma, vol. 206, pp. 49-62. DOI 10.1016/j.geoderma.2013.04.011.

TAGUAS, E. V., GUZMÁN, E., GUZMÁN, G., VANWALLEGHEM, T. y GÓMEZ, J.A., 2015. Characteristics and importance of rill and gully erosion: a case study in a small catchment of a marginal olive grove. Cuadernos de Investigación Geográfica, vol. 41, no. 1, pp. 107-126. DOI 10.18172/cig.2644.

TAGUAS, E. V., PEÑA, A., AYUSO, J.L., PÉREZ, R., YUAN, Y. y GIRÁLDEZ, J.V., 2010. Rainfall variability and hydrological and erosive response of an olive tree microcatchment under no-tillage with a spontaneous grass cover in Spain. Earth Surface Processes and Landforms, vol. 35, no. 7, pp. 750760. DOI 10.1002/esp.1893. 
TAGUAS, E.V., YUAN, Y., BINGNER, R.L. y GÓMEZ, J.A., 2012. Modeling the contribution of ephemeral gully erosion under different soil managements: A case study in an olive orchard microcatchment using the AnnAGNPS model. CATENA, vol. 98, pp. 1-16. ISSN 0341-8162. DOI 10.1016/j. catena.2012.06.002.

TAGUAS, Encarnación V., YUAN, Y., LICCIARDELLO, F. y GÓMEZ, J.A., 2015. Curve numbers for olive orchard catchments: case study in Southern Spain. Journal of Irrigation and Drainage Engineering, vol. 141, no. 11, pp. 05015003. ISSN 0733-9437. DOI 10.1061/(ASCE)IR.1943-4774.0000892.

VANWALLEGHEM, T., LAGUNA, A., GIRÁLDEZ, J.V. y JIMÉNEZ-HORNERO, F.J., 2010. Applying a simple methodology to assess historical soil erosion in olive orchards. Geomorphology, vol. 114, no. 3 , pp. 294-302. ISSN 0169-555X. DOI 10.1016/j.geomorph.2009.07.010.

WIRTZ, S., ISERLOH, T., ROCK, G., HANSEN, R., MARZEN, M., SEEGER, M., BETZ, S., REMKE, A., WENGEL, R., BUTZEN, V. y OTHERS, 2012. Soil erosion on abandoned land in Andalusia: A comparison of interrill-and rill erosion rates. ISRN Soil Science, vol. 2012. DOI 10.5402/2012/730870.

WIRTZ, S., SEEGER, M. y RIES, J.B., 2010. The rill experiment as a method to approach a quantification of rill erosion process activity. Zeitschrift für Geomorphologie, NF, vol. 54, no. 1, pp. 47-64.

WIRTZ, S., SEEGER, M. y RIES, J.B., 2012. Field experiments for understanding and quantification of rill erosion processes. Catena, vol. 91, pp. 21-34. ISSN 0341-8162. DOI http://dx.doi.org/10.1016/j. catena.2010.12.002.

WIRTZ, S., SEEGER, M., ZELL, A., WAGNER, C., WAGNER, J.-F. y RIES, J.B., 2013. Applicability of different hydraulic parameters to describe soil detachment in eroding rills. PLOS ONE, vol. 8, no. 5, pp. 1-11. DOI 10.1371/journal.pone.0064861.

YU, Y., LOISKANDL, W., KAUL, H.-P., HIMMELBAUER, M., WEI, W., CHEN, L. y BODNER, G., 2016. Estimation of runoff mitigation by morphologically different cover crop root systems. Journal of Hydrology, vol. 538, pp. 667-676. ISSN 0022-1694. DOI 10.1016/j.jhydrol.2016.04.060.

ZEMA D. A., DENISI P., TAGUAS RUIZ E. V., GÓMEZ J. A., BOMBINO G. y FORTUGNO D., 2015. Evaluation of Surface Runoff Prediction by AnnAGNPS Model in a Large Mediterranean Watershed Covered by Olive Groves. Land Degradation \& Development, vol. 27, no. 3, pp. 811-822. DOI 10.1002/ Idr.2390. 